\title{
SYNTHESIS, CHARACTERIZATION AND ANTIMICROBIAL SCREENING OF 2-(2-ARYLIDENEHYDRAZINYL)-4-(3- CHLOROPHENYL)-6-HYDROXYPYRIMIDINE-5- CARBONITRILE
}

\author{
Pravin T. Tryambake* \\ Department of Chemistry, S.N. Arts, D. J. M. Commerce \& B. N. S. Science College, \\ Sangamner, Ahmednagar-422 605, India \\ *E-mail: pravintryambake21@ rediffmail.com
}

\begin{abstract}
A series of novel 2-(2-arylidenehydrazinyl)-4-(3-chlorophenyl)-6-hydroxypyrimidine-5-carbonitrile (4a-f) were synthesized from the reactions of 4-(3-chlorophenyl)-6-hydroxy-2-mercaptopyrimidine-5carbonitrile. The newly synthesized compounds were characterized using by IR, ${ }^{1} \mathrm{H}-\mathrm{NMR}$, Mass spectroscopy and elemental analysis. In addition, the synthesized compounds were screened for their in vitro antibacterial and antifungal activities.
\end{abstract}

Keywords: Pyrimidine, antibacterial, antifungal activity.

(c) RASĀYAN. All rights reserved

\section{INTRODUCTION}

Pyrimidine is a six-member organic heterocyclic compound consisting of two nitrogen atoms in the ring at 1 and 3 positions. Many pyrimidine derivatives exhibit a broad spectrum of biological activities such as antimicrobial, ${ }^{1}$ anti-inflammatory, ${ }^{2,3}$ antitumor, ${ }^{4-5}$ CXCR2 receptor antagonists, ${ }^{6}$ analgesic activities, ${ }^{7-8}$ antineoplastic agents, ${ }^{9}$ renin inhibitors, ${ }^{10}$ anticancer. ${ }^{11}$

In view of applications of pyrimidine derivatives in the clinical field, we report the synthesis of a series of 2-(2-arylidenehydrazinyl)-4-(3-chlorophenyl)-6-hydroxypyrimidine - 5-carbonitrile (4a-f). Synthesis of target compounds has been done by condensation of various aromatic aldehydes with 4-(3-chlorophenyl)2-hydrazinyl-6-hydroxypyrimidine-5-carbonitrile (3). The structures of the synthesized products were confirmed by the spectral (IR, ${ }^{1} \mathrm{H}$ NMR and Mass-4b and 4c) and elemental analysis.

\section{EXPERIMENTAL}

Melting points were measured in open capillary tubes and are uncorrected. The progress of the reaction was monitored by thin layer chromatography (TLC) on Merck silica gel $60 \mathrm{~F}_{254}$ aluminum sheets and visualized by UV light. IR spectra were recorded on Shimadzu FTIR (KBr) - 408 spectrophotometers. The ${ }^{1} \mathrm{H}$ NMR spectra were recorded at $400 \mathrm{MHz}$ in DMSO- $\mathrm{d}_{6}$ using TMS as internal standard and are given in $\delta$ units. The LC-MS spectra were recorded on WATER, Q-TOF Micro mass. The synthesized compounds were named using ChemDraw Ultra software (v.12.0). Compound 1 was prepared from the reaction of 3-chlorobenzaldehyde, ethyl cyanoacetate, and thiourea as per reported method. ${ }^{4,}{ }^{12}$ Further compounds $\mathbf{1}$ was converted into $\mathbf{3}$ as described in literature. ${ }^{13,14}$

Synthesis of ethyl 2-(4-(3-chlorophenyl)-5-cyano-6-hydroxypyrimidin-2-ylthio) acetate (2)

Compound 1 ( $1 \mathrm{mmol})$ was dissolved in $15 \mathrm{ml}$ DMF. To this solution ethyl chloroacetate $(1 \mathrm{mmol})$ was added dropwise and a catalytic amount of triethylamine. The reaction mixture was stirred at for $7 \mathrm{~h}$ until the reaction was completed monitored by TLC. The reaction mixture was poured into ice-cold water. 
Separated solid was filtered, washed with water, dried. The residue obtained was purified by recrystallization from ethanol. The structure of compound 2 was confirmed by IR and ${ }^{1} \mathrm{H}$ NMR.

Yield: $87 \%$, mp216-218 ${ }^{\circ} \mathrm{C} . \mathrm{IR}(\mathrm{KBr}) \mathrm{cm}^{-1}: 3453,3301$ (enolicOH), 2942(CH aliphatic), $2222(-\mathrm{CN})$, 1734 ( $\mathrm{C}=\mathrm{O}$ ester), 1665 ( $\mathrm{C}=\mathrm{O}$ pyrimidine), 1251 (C-O-C), 884 (C-S-C), 780 (Ar-Cl); 1H-NMR (400MHz, DMSO-d 6 ,ppm): 1.13(t, J=7.12, 3H, $\left.-\mathrm{CH}_{3}\right), 4.11\left(\mathrm{t}, \mathrm{J}=7.12,2 \mathrm{H},-\mathrm{OCH}_{2}\right), 4.07\left(\mathrm{~s}, 2 \mathrm{H},-\mathrm{SCH}_{2}\right)$, 7.58 (t, 1H, Ar-H), 7.66 (d, 1H, Ar-H), 7.88(d, 1H, Ar-H), 7.89 (s, 1H, Ar-H).

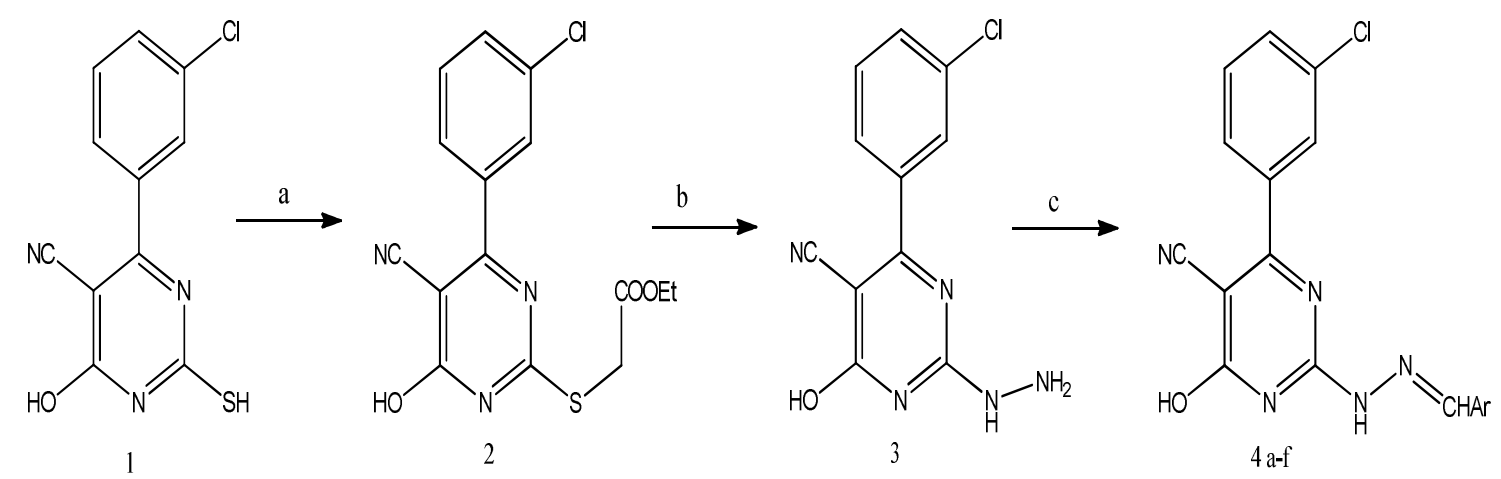

(a) $\mathrm{ClCH}_{2} \mathrm{COOEt} / \mathrm{K}_{2} \mathrm{CO}_{3} / \mathrm{DMF}$ rt (b) Hydrazine hydrate/ Methanol reflux

(c)aromatic aldehyde/ $\mathrm{AcOH} / \mathrm{EtOH}$ reflux.

Scheme-1

Synthesis of 4-(3-chlorophenyl)-2-hydrazinyl-6-hydroxypyrimidine-5-carbonitrile (3)

Compound $2(1 \mathrm{mmol})$ was dissolved in $20 \mathrm{ml}$ ethanol. To this solution hydrazine hydrate $(1.1 \mathrm{mmol})$ was added. The reaction mixture was refluxed for $4 \mathrm{~h}$ until the reaction was completed monitored by TLC. After completion of the reaction, the contents were cooled to room temperature. The separated solid was filtered washed with ethanol,dried and used for next reaction. Yield: $83 \%$. M.p. $260-262{ }^{\circ} \mathrm{C}$. IR (KBr) $\mathrm{cm}^{-1}$ : 3501- 3301 (enolic $\mathrm{OH}, \mathrm{NH} \& \mathrm{NH}_{2}$ ), 2206 (-CN), 780 (Ar-Cl).

Synthesis of 2-(2-arylidenehydrazinyl)-4-(3-chlorophenyl)-6-hydroxypyrimidine-5-carbonitrile (4af)

A mixture of Compound $3(1 \mathrm{mmol})$, aromatic aldehyde $(1 \mathrm{mmol})$, and the glacial acetic acid catalytic amount in absolute ethanol $(15 \mathrm{ml})$ was refluxed for $4 \mathrm{~h}$. A formed precipitate was filtered off, washed with water and dried. The residue obtained was purified by silica gel column chromatography.

2-((E)-2-(4-hydroxybenzylidene) carbonitrile (4a)

Yield $88 \%$. m. p. $250^{\circ} \mathrm{C} . \mathrm{IR}(\mathrm{KBr}) \mathrm{cm}^{-1}: 3514$ (enolic OH), 3399(OH), 3241(NH), 3072( Ar-H), 2215(CN), 1680(-HC=N-), 777 (Ar-Cl). ${ }^{1} \mathrm{H}-\mathrm{NMR}:$ 6.88(d, J=8.6, 2H, Ar-H), 7.63(t, J=7.84, 1H, Ar-H), 7.71(d, 1H, Ar), $7.88(\mathrm{~d}, 2 \mathrm{H}, \mathrm{Ar}-\mathrm{H}), 7.92(\mathrm{~d}, 2 \mathrm{H}, \mathrm{Ar}-\mathrm{H}), 8.13(\mathrm{~s}, 1 \mathrm{H}, \mathrm{Ar} \mathrm{N}=\mathrm{CH}), \quad 10.07$ (s, 1H, Ar-OH), 12.36(s, 1H, NH), 12.52( s, 1H, Py-OH).

2-((E)-2-(4-hydroxy 3-methoxybenzylidene) hydrazinyl)-4-(3-chlorophenyl)-6-hydroxypyrimidine5-carbonitrile (4b)

Yield: $90 \%$, m.p. $320{ }^{\circ} \mathrm{C}$. IR (KBr) cm ${ }^{-1}: 3530(\mathrm{OH}), 3252($ enolic $\mathrm{OH}), 3216(\mathrm{NH}), 3070(\mathrm{Ar}-\mathrm{H})$, 2208(CN), $1670(-\mathrm{HC}=\mathrm{N}-), 780(\mathrm{Ar}-\mathrm{Cl}) .{ }^{1} \mathrm{H}-\mathrm{NMR}: 3.88\left(\mathrm{~s}, 3 \mathrm{H}, \mathrm{OCH}_{3}\right) 6.83(\mathrm{~d}, \mathrm{~J}=8.12,2 \mathrm{H}, \mathrm{Ar}-\mathrm{H})$, 7.27(dq, J=6.84, 1H, Ar-H), 7.58(t, J=7.8, 1H, Ar-H), 7.66 (d, 1H, J=7.8, Ar-H), 7.70 (s, 1H, Ar-H), 8.08(s, 1H, Ar N=CH), 9.64 (s, 1H, Ar-OH), 12.19(s, 1H, NH) 12.49( s, 1H, Py-OH). LC-MS, m/z: $396.29(\mathrm{M}+1)$. 


\section{RASĀYAN J. Chem.}

Vol. 11 | No. 1 |69-73 | January - March | 2018

2-((E)-2-(2-fluorobenzylidene) hydrazinyl)-4-(3-chlorophenyl)-6-hydroxypyrimidine-5-carbonitrile (4c)

Yield 88\%. m.p. $326^{\circ}$ C.IR (KBr) cm ${ }^{-1}$ : 3466 (enolic OH), 3204( NH), 3022( Ar-H), 2211(CN), 1656 ($\mathrm{HC}=\mathrm{N}-), 776(\mathrm{Ar}-\mathrm{Cl}) .1 \mathrm{H}-\mathrm{NMR}: 3.88\left(\mathrm{~s}, 3 \mathrm{H}, \mathrm{OCH}_{3}\right)$ 6.83(d, J=8.12, 2H, Ar-H), 7.27(dq, J=6.84, 1H, Ar$\mathrm{H}), 7.58(\mathrm{t}, \mathrm{J}=7.8,1 \mathrm{H}, \mathrm{Ar}-\mathrm{H}), 7.66(\mathrm{~d}, 1 \mathrm{H}, \mathrm{J}=7.8, \operatorname{Ar}-\mathrm{H}), 7.70(\mathrm{~s}, 1 \mathrm{H}, \mathrm{Ar}-\mathrm{H}), 8.08(\mathrm{~s}, 1 \mathrm{H}, \mathrm{Ar} \mathrm{N}=\mathrm{CH})$, 12.48( br s, 2H, NH, Py-OH). LC-MS, m/z: $368.31(\mathrm{M}+1)$.

\section{2-((E)-2-(3-hydroxybenzylidene)}

carbonitrile (4d)

Yield 92\%. m.p. $310{ }^{\circ} \mathrm{C} . \mathrm{IR}(\mathrm{KBr}) \mathrm{cm}^{-1}: 3467$ (enolic OH), $3210(\mathrm{NH}), 3068(\mathrm{Ar}-\mathrm{H}), 2208(\mathrm{CN}), 1656(-$ $\mathrm{HC}=\mathrm{N}-), 779(\mathrm{Ar}-\mathrm{Cl}) .{ }^{1} \mathrm{H}-\mathrm{NMR}: 6.87(\mathrm{dd}, \mathrm{J}=6.16$ and 1.76, $1 \mathrm{H}, \mathrm{Ar}-\mathrm{H}), 7.22(\mathrm{t}, \mathrm{J}=7.88,1 \mathrm{H}, \mathrm{Ar}-\mathrm{H}), 7.35(\mathrm{~d}$, $\mathrm{J}=7.64,1 \mathrm{H}, \mathrm{Ar}-\mathrm{H}), 7.45$ (s, 1H, Ar-H), 7.51-7.59 (m, 2H, Ar-H), 7.84-7.87(m, 2H, Ar H), 9.43 (s, 1H, Ar$\mathrm{OH}), 12.40$ ( s, 2H, Py-OH, NH).

2-((E)-2-((furan-2-yl)methylene)hydrazinyl)-4-(3-chlorophenyl)-6-hydroxypyrimidine-5carbonitrile $(4 \mathrm{e})$

Yield: 90\%. m.p. $300{ }^{\circ} \mathrm{C} . \mathrm{IR}(\mathrm{KBr}) \mathrm{cm}^{-1}$ : 3462 (enolic OH), $3221(\mathrm{NH}), 3042(\mathrm{Ar}-\mathrm{H}), 2208(\mathrm{CN}), 1667$ ($\mathrm{HC}=\mathrm{N}-), 791(\mathrm{Ar}-\mathrm{Cl}) .{ }^{1} \mathrm{H}-\mathrm{NMR}: 6.60(\mathrm{t}, \mathrm{J}=1.72,1 \mathrm{H}, \mathrm{Ar}-\mathrm{H}), 7.20(\mathrm{~d}, \mathrm{~J}=3.36,1 \mathrm{H}, \mathrm{Ar}-\mathrm{H}), 7.58(\mathrm{~m}, 2 \mathrm{H}, \mathrm{Ar}-\mathrm{H})$, $7.75(\mathrm{~d}, 1 \mathrm{H}, \mathrm{J}=1.32, \mathrm{Ar}-\mathrm{H}), 7.86(\mathrm{~m}, 2 \mathrm{H}, \mathrm{Ar}-\mathrm{H}), 8.16(\mathrm{~s}, 1 \mathrm{H}, \mathrm{Ar} \mathrm{N}=\mathrm{CH}), \quad 11.83(\mathrm{~s}, 1 \mathrm{H}, \mathrm{NH}) 12.65(\mathrm{~s}, 1 \mathrm{H}$, $\mathrm{Py}-\mathrm{OH})$.

2-((E)-2-(3,4,5-trimethoxybenzylidene) hydrazinyl)-4-(3-chlorophenyl)-6-hydroxypyrimidine-5carbonitrile (4f)

Yield 90\%. m.p. $320^{\circ} \mathrm{C} . \mathrm{IR}(\mathrm{KBr}) \mathrm{cm}^{-1}: 3467$ (enolic OH), $3220(\mathrm{NH}), 3043(\mathrm{Ar}-\mathrm{H}), 2208(\mathrm{CN}), 1656$ ($\mathrm{HC}=\mathrm{N}-), 779(\mathrm{Ar}-\mathrm{Cl})$

${ }^{1} \mathrm{H}-\mathrm{NMR}: 3.74\left(\mathrm{~s}, 3 \mathrm{H}, \mathrm{OCH}_{3}\right), 3.88\left(\mathrm{~s} 6 \mathrm{H}, \mathrm{OCH}_{3}\right), 7.26(\mathrm{~s}, 2 \mathrm{H}, \mathrm{Ar}-\mathrm{H}), 7.61(\mathrm{~m}, 2 \mathrm{H}, \mathrm{Ar}-\mathrm{H}), 7.8(\mathrm{~m}, 2 \mathrm{H}, \mathrm{Ar}-$ $\mathrm{H}), 8.11(\mathrm{~s}, 1 \mathrm{H}, \mathrm{Ar} \mathrm{N}=\mathrm{CH}), \quad 12.65(\mathrm{~s}, 2 \mathrm{H}, \mathrm{Py}-\mathrm{OH}, \mathrm{NH})$.

Table-1: Synthesis of 2-(2-arylidenehydrazinyl)-4-(3-chlorophenyl)-6-hydroxypyrimidine-5-carbonitrile (4a- f)

\begin{tabular}{c|c|c|c|c}
\hline Entry & R-CHO & $\begin{array}{c}\text { Yield }^{\mathrm{a}} \\
(\%)\end{array}$ & M.P. $\left({ }^{\circ} \mathrm{C}\right)$ & $\begin{array}{c}\text { Comp. } \\
\text { no. }\end{array}$ \\
\hline 1 & $4-\mathrm{OH} \mathrm{C} \mathrm{H}_{4}$ & 88 & 250 & $4 \mathrm{a}$ \\
\hline 2 & $4-\mathrm{OH}, 3-\mathrm{OCH}_{3} \mathrm{C}_{6} \mathrm{H}_{4}$ & 90 & 320 & $4 \mathrm{~b}$ \\
\hline 3 & $4-\mathrm{F} \mathrm{C}_{6} \mathrm{H}_{4}$ & 88 & 326 & $4 \mathrm{c}$ \\
\hline 4 & $3-\mathrm{OH} \mathrm{C}_{6} \mathrm{H}_{4}$ & 92 & 310 & $4 \mathrm{~d}$ \\
\hline 5 & Furyl & 90 & 300 & $4 \mathrm{e}$ \\
\hline 6 & $3,4,5-\left(\mathrm{OCH}_{3}\right)_{3} \mathrm{C}_{6} \mathrm{H}_{2}$ & 90 & 320 & $4 \mathrm{f}$ \\
\hline
\end{tabular}

\section{Antimicrobial Activity}

Synthesized compounds (4a-f) were evaluated for their antibacterial activity against Escherichia coli, Pseudomonas aeruginosa (two-gram negative) and Staphylococcus aureus, Streptococcus pyogenes (two gram-positive) using Broth dilution method. ${ }^{15}$ Ampicillin used as a standard drug, the MIC results are represented in Table-2. Synthesized compounds (4a-f) also tested for their antifungal activity against three fungi Candida albicans, Aspergergillus niger, Aspergergillusclavatus, greseofulvin used as standard drug. The MIC results are represented in Table-3.

\section{RESULTS AND DISCUSSION}

Initially 6-(3-chlorophenyl) -4-oxo- 2-thioxo - 1,2,3,4-tetrahydropyrimidine-5-carbonitrile (1) was synthesized as per reported method. Compound 1 on reaction with ethyl chloroacetate was converted to ethyl 2-((4-(3-chlorophenyl)-5-cyano-6-oxo-1,6-dihydropyrimidin-2-yl)thio)acetate (2). Further compound 2 was treated with hydrazine hydrate under the refluxed condition and converted to 2- 
hydrazinyl-4-(3-chlorophenyl)-6-oxo-1,6-dihydropyrimidine-5-carbonitrile (3). Finally, a series of 2-(2arylidenehydrazinyl)-4-(3-chlorophenyl)-6-oxo-1,6-dihydro pyrimidine-5-carbonitrile (4a-f) was synthesized by condensation of compound 3 with various aromatic aldehydes. The formation of target compounds (4a-f) was supported by spectral analysis. The FT-IR showed absorption bands in the range of $3530-3463 \mathrm{~cm}^{-1}$ for enolic $-\mathrm{OH}, 3204-3316 \mathrm{~cm}^{-1}$ for $-\mathrm{NH}-, \quad 2950-3070 \mathrm{~cm}^{-1}$ for aromatic $\mathrm{C}-\mathrm{H}, 2208-2220$ $\mathrm{cm}^{-1}$ for $-\mathrm{CN}, 1600-1670 \mathrm{~cm}^{-1}$ for azomethine group $(-\mathrm{CH}=\mathrm{N}-)$ and $770-780 \mathrm{~cm}^{-1}$ for Ar-Cl. The ${ }^{1} \mathrm{H}$ NMR spectra of title compounds (4a-h) showed peaks of enolic $-\mathrm{OH}$, hydrazide $(\mathrm{NH})$, aromatic, imine ($\mathrm{CN}=\mathrm{N}-$ ) proton. The ${ }^{1} \mathrm{H}$ NMR spectra of compounds (4a-f) showed broad singlet at $\delta 11.83-12.65$ indicating the presence of hydrazide $-\mathrm{NH}-$ and enolic $-\mathrm{OH}$. The sharp singlet around $\delta 8.10$ indicating the presence of azomethine $(-\mathrm{CH}=\mathrm{N}-)$ proton. The appearance of signals in the range of 6.8-7.8 was due to aromatic protons. In the mass spectrum compound, 4b showed a peak at $\mathrm{m} / \mathrm{z} 396.29(\mathrm{M}+1)$ and compound $4 \mathrm{c}$ showed a peak at $\mathrm{m} / \mathrm{z} 368.31(\mathrm{M}+1)$. All the newly synthesized compounds $4 \mathrm{a}-\mathrm{f}$ were screened in vitro for their antimicrobial activities. The MIC values $(\mu \mathrm{g} / \mathrm{ml})$ of $4 \mathrm{a}-\mathrm{f}$ along with standard drugs against selected microbes are shown in Table-2 and 3. All compounds 4a-f shows mild to moderate antibacterial activity against selected microbes as compared to standard drug chloramphenicol.

Table 2: In vitro antibacterial activities of compounds (4a- f)(Minimum inhibition concentration, $\mu \mathrm{g} / \mathrm{ml})$

\begin{tabular}{c|c|c|c|c}
\hline S.No. & E. coli & P. aeruginosa & S. aureus & S. pyogenus \\
\hline $4 \mathrm{a}$ & 500 & 200 & 125 & 200 \\
\hline $4 \mathrm{~b}$ & 500 & 500 & 200 & 62.5 \\
\hline $4 \mathrm{c}$ & 100 & 250 & 250 & 100 \\
\hline $4 \mathrm{~d}$ & 200 & 125 & 125 & 100 \\
\hline $4 \mathrm{e}$ & 100 & 125 & 200 & 200 \\
\hline $4 \mathrm{f}$ & 62.5 & 100 & 125 & 250 \\
\hline AMPICILLIN & 100 & -- & 250 & 100 \\
\hline CHLORAMPHENICOL & 50 & 50 & 50 & 50 \\
\hline
\end{tabular}

Table 3: In vitro antifungal activities of compounds (4a- f)

(Minimum inhibition concentration, $\mu \mathrm{g} / \mathrm{ml}$ )

\begin{tabular}{c|c|c|c}
\hline Compounds & C. albicans & A. niger & A. clavatus \\
\hline $4 \mathrm{a}$ & 250 & 500 & 500 \\
\hline $4 \mathrm{~b}$ & 1000 & 1000 & 1000 \\
\hline $4 \mathrm{c}$ & 1000 & 250 & 250 \\
\hline $4 \mathrm{~d}$ & 1000 & 1000 & 1000 \\
\hline $4 \mathrm{e}$ & 500 & $>1000$ & $>1000$ \\
\hline $4 \mathrm{f}$ & 250 & 500 & 500 \\
\hline NYSTATIN & 100 & 100 & 100 \\
\hline GRESEOFULVIN & 500 & 100 & 100 \\
\hline
\end{tabular}

But as compared to another standard drug ampicillin all synthesized compounds show good for mild activity. Compounds 4c, 4e, 4f shows promising activity against E. coli, compounds 4d, 4e, 4f shows good activity against $\mathrm{P}$. aeruginosa, compounds $4 \mathrm{a}, 4 \mathrm{~d}$, $4 \mathrm{f}$ shows good activity against $\mathrm{S}$. Aureus and compounds $4 \mathrm{~b}, 4 \mathrm{c}, 4 \mathrm{~d}$ shows good activity against $\mathrm{S}$. pyogenus. Out of which $4 \mathrm{f}$ exhibited most promising activity against E. coli that is $62.5 \mu \mathrm{g} / \mathrm{ml}$ and $4 \mathrm{~b}$ exhibited most promising activity against $\mathrm{S}$. pyogenus that is $62.5 \mu \mathrm{g} / \mathrm{ml}$. Microbial activity data showed that compound $4 \mathrm{a}$, 4b showed mild antibacterial activity $(>200 \mu \mathrm{g} / \mathrm{ml})$ against E. coli, compounds $4 \mathrm{a}, 4 \mathrm{~b}$, and $4 \mathrm{c}$ exhibited mild antibacterial $(>200 \mu \mathrm{g} / \mathrm{ml})$ against P. aeruginosa,compounds $4 \mathrm{~b}, 4 \mathrm{c}$ and $4 \mathrm{e}$ exhibited mild antibacterial $(>200 \mu \mathrm{g} / \mathrm{ml})$ 
RASĀYAN J. Chem.

Vol. 11 | No. 1 |69-73 | January - March | 2018

against S. Aureus and compounds 4a, 4e and 4f exhibited mild antibacterial $((>200 \mu \mathrm{g} / \mathrm{ml})$ against S. pyogenus as compared to standard drug Ampicilin $(100 \mu \mathrm{g} / \mathrm{ml})$. All compounds 4a-f shows mild to moderate antifungal activity against selected microbes as compared to standard drug greseofulvin. Compounds $4 \mathrm{a}, 4 \mathrm{e}, 4 \mathrm{f}$ exhibit good antifungal activity against $\mathrm{C}$. albicans and other compounds shows mild antifungal activity.

\section{CONCLUSION}

In present study, design, and synthesis of a new pyrimidine, 2-(2-arylidenehydrazinyl)-4-(3chlorophenyl)-6-oxo-1,6-dihydro pyrim-idine-5-carbonitrile (4a-h) derivatives are described. All the derivatives were characterized by physical and spectral analysis. All the synthesized compounds were evaluated for their antibacterial and antifungal activities. It was noted that the most of the derivatives were show mild to moderate and good antibacterial and antifungal activities. As per result of antibacterial activity compounds $4 \mathrm{c}, 4 \mathrm{e}$ and $4 \mathrm{f}$ show most promising activity while $4 \mathrm{~b}$ shows excellent activity against $\mathrm{S}$. pyogenus. Compound s $4 \mathrm{a}, 4 \mathrm{c}$ and $4 \mathrm{f}$ shows have remarkable antifungal activity.

\section{ACKNOWLEDGEMENT}

The author is grateful to BCUD, Savitribai Phule Pune University, Pune (Grant No. OSD/BCUD/360/151) for financial assistance and Director, SAIF, Panjab University, Chandigarh for providing spectral analysis facility. The author also thankful to Principal, S.N. Arts, D. J. M. Commerce \& B. N. S. Science College, Sangamner.

\section{REFERENCES}

1. A. Nagaraj, C. S.Reddy, Iran. Chem. Soc., 5, 262 (2008)

2. M. Sham, N.S. Sondhi, J. Monika, A. Kumar, Bioorg. Med. Chem., 13, 6158 (2005)

3. H. N.Hafez, A.- R. B.A.El-Gazzar, G. A.M.Nawwar, Eur. J. of Med. Chem., 45, 1485 (2010)

4. H.T. Abdel-Mohsen, F. A. F. Ragab, M. M. Ramla and H. I. EI. Diwani, Eur. J. of Med. Chem., 45, 2336 (2010).

5. M. T.Cocco, C. Congiu, V. Onnis, R. Piras, Il Farmaco, 56, 741 (2001)

6. D. W. Porter, M. Bradley, Z. Brown, S. J. Charlton, B. Cox, P. Hunt, D. Janus, S. Lewis, P. Oakley, D. O’Connor, J. Reilly, N. Smith and N. J. Press, Bioorg. \& Med. Chem. Lett., 24, 3285(2014)

7. A. Vidal, M. L. Ferrandiz, A. Ubeda, A. Acero-Alarcon, J. Sepulveda-Arques and M.J. Alcaraz, J. Pharm. Pharmacol., 53, 1379(2001)

8. S.M. Sondhi, S. Rajvanshi, N. Singh, S. Jain and A. K. Lahoti, Central Eur. J. Chem., 2, 141(2004).

9. E. Menta, M. Palumbo, Expert Opin.Therap. Patents, 7, 1401(1997).

10. Y. Imaeda, M. Tawada, S. Suzuki, M. Tomimoto, M. Kondo,N. Tarui, T. Sanada, R. Kanagawa, G. Snell, C. A. Behnke, K. Kubo, T. Kuroita, Bioorg. Med. Chem., 24, 5771 (2016).

11. O.A. Fathalla, I.F. Zeid, M.E. Haiba, A.M. Soliman, S. I. Abd-Elmoez, W.S. El-Serwy, World Journal of Chemistry, 4,127(2009)

12. S. Kambe, K. Saito, H. Kishi, Synthesis, 4, 287(1979)

13. D. N.Upadhyay, V. J. Ram, Indian J. of Chem., 38B, 173(1999)

14. S. Kotaiah, D. V. Reddy, B. Ramadevi, A. Naidu, P. K. Dubey, Hetero. Letters., 4, 335(2014).

15. I. Wiegand, K. Hilpertand R. E. W. Hancock, Nature Protocols, 3, 163(2008).

[RJC-1615/2017] 